\title{
Das Institut für Sächsische Geschichte und Volkskunde e. V. in Dresden Bericht für das Jahr 2015
}

von

ENNO BÜNZ

Mit drei gemeinsamen Forschungsvorhaben der Bereiche Geschichte und Volkskunde, acht Vorhaben des Bereichs Geschichte und zwölf Projekten des Bereichs Volkskunde wird neuerlich deutlich, dass das ISGV auch in diesem Berichtsjahr seine Arbeit erfolgreich und mit einem breiten Themenspektrum aus der Geschichte und Volkskunde Sachsens fortgesetzt hat. Vergleichende und grenzübergreifende Perspektiven in die Nachbarländer, Kooperationen mit zahlreichen wissenschaftlichen Institutionen inner- wie außerhalb Sachsens sind dabei seit langem selbstverständlich und verdeutlichen das Ansehen, welches sich das ISGV seit seiner Gründung 1997 erworben hat.

In bewährter Weise ergänzen sich dabei langfristig angelegte Grundlagenforschung, die in beiden Bereichen das wichtigste Standbein des Instituts ist, und mittelbzw. kurzfristig angelegte Projekte. Das gemeinsame Vorhaben „Sachsen: weltoffen!“ verdeutlicht, wie beides auch ineinandergreifen kann, beschäftigen sich Mitarbeiter des ISGV doch seit geraumer Zeit in verschiedenen Teilprojekten mit Themen der Migration, sodass sich das Institut nun auch fundiert in aktuelle Debatten um Zuwanderung nach Sachsen einbringen kann und mit einer vertieften historischen Perspektive zur Versachlichung der Debatte beitragen möchte.

Aus den vielfältigen Arbeiten im Bereich Geschichte ist an dieser Stelle Folgendes hervorzuheben: Das Dauervorhaben der "Sächsischen Biografie“ hat die gewohnt guten Fortschritte gemacht. Das Editionsvorhaben der Briefe der Herzogin Elisabeth von Sachsen (Fürstinnenkorrespondenzen der Reformationszeit) hat mit dem Abschluss des zweiten Bandes ein wichtiges Zwischenziel erreicht und stellt einen essenziellen Beitrag zum 2017 anstehenden Reformationsjubiläum dar. Das Promotionsvorhaben „Für Gott und Vaterland - Patriotismus und Militärdienst in Sachsen 1806 bis 1866/67“ steht vor dem baldigen Abschluss.

Für den Bereich Volkskunde ist festzuhalten, dass die Langzeitvorhaben „Lebensgeschichtliches Archiv für Sachsen“ und „Visuelle Quellen zur Volkskultur in Sachsen“ kontinuierlich ausgebaut wurden. Gute Fortschritte konnten auch im Projekt „Zwischen Aufstieg und Krise. Städtische Identität und Selbstwahrnehmung in Plauen 1880 bis 1933 “ erzielt werden, dessen Ergebnisse in eine monografische Darstellung einfließen werden. Neu angelaufen ist das Drittmittelprojekt „Kontaktzonen. Kulturelle Praktiken im deutsch-tschechisch-polnischen Grenzraum“. Die Erforschung der Brigadebücher als Zeugnisse der Arbeitskultur in der DDR wird seit diesem Jahr im Rahmen eines Promotionsvorhabens unter dem Titel „Erinnern an die Arbeit im Kollektiv“ fortgeführt.

Im Berichtsjahr wurden von beiden Bereichen fünf Tagungen durchgeführt, und zwar über Arbeiterfotografie der Weimarer Republik (in Dresden), Kurfürst August von Sachsen (Torgau), die Elbe (Hamburg), die Pfalzgrafen bei Rhein und die Herzöge von Sachsen im Vergleich (Dresden) und die Merinoschafzucht im 18./19. Jahrhundert (Dresden). Die Beteiligung an mehreren Ausstellungsprojekten ist hier ebenfalls her- 
vorzuheben. Diese Vorhaben verdeutlichen erneut die Vernetzung des ISGV, das mittlerweile mit einer Fülle von Kooperationspartnern in Sachsen und darüber hinaus zusammenarbeitet und als wissenschaftlicher Partner auch in dieser Hinsicht gefragt ist.

In den „Schriften zur sächsischen Geschichte und Volkskunde“ konnten im Berichtsjahr vier Bände vorgelegt und mit der Herausgabe des 50. Bandes eine markante Zäsur erreicht werden. In der Reihe der „Bausteine aus dem ISGV“ liegen nun 33 Bände vor. Die „Quellen und Materialien zur sächsischen Geschichte und Volkskunde" konnten im Berichtsjahr mit einem gewichtigen fünften Band fortgesetzt werden. Die Reihe „Spurensuche“ umfasst ebenfalls fünf Bände. Außerdem sind die beiden Zeitschriften des Instituts - das „Neue Archiv für sächsische Geschichte“ und die „Volkskunde in Sachsen“ - im gewohnten jährlichen Rhythmus erschienen. Neben den Redaktionsarbeiten an den gedruckten Veröffentlichungen hat auch die laufende Betreuung der Internet-Publikationen, nunmehr insgesamt zehn Vorhaben, im Berichtszeitraum wieder viel Arbeitskraft gebunden. Zudem wurden fünf Newsletter veröffentlicht.

Die ISGV-Homepage und die Projektseiten wurden im Berichtszeitraum von 16123/118 334 Besuchern mit 95 650/704004 Seitenaufrufen frequentiert. Im dritten Quartal 2015 konnte die neue ISGV-Homepage online geschaltet werden. Anschließend begann die Gestaltung der Webseiten für die einzelnen Online-Projekte.

Die Finanzierung bleibt nach mehrfacher Überrollung des Doppelhaushaltes schwierig und wird bei zu befürchtender weiterer Verschärfung die Arbeitsfähigkeit des Instituts nachhaltig beeinträchtigen, da vakante Stellen nicht sofort wiederbesetzt werden können und keine Mittel zur Verfügung stehen, um Drittmittelanträge ausarbeiten zu lassen. Da viele Projekte des Instituts mittlerweile im Internet präsent sind, bedroht auch eine veraltende EDV-Ausstattung, die nicht erneuert werden kann, die Wirkungsmöglichkeiten des Instituts gravierend. Trotz dieser wachsenden Schwierigkeiten, die sich abzeichnen, konnten im Berichtszeitraum die laufenden Projekte weiter fortgesetzt werden.

Das Institut und seine Mitarbeiter haben sich auch durch Tagungen und Vorträge, Lehrtätigkeit an Universitäten, Beratungstätigkeit in Gremien und Kommissionen der interessierten Öffentlichkeit präsentiert. Angesichts des insgesamt begrenzten finanziellen und personellen Rahmens verrichtet das ISGV als Dienstleister im Land und darüber hinaus eine viel beachtete Arbeit, die auch von den die Institutsarbeit begleitenden Gremien positiv anerkannt wird.

\section{Forschungsprojekte 2015}

\section{Gemeinsame Projekte der Bereiche Geschichte und Volkskunde}

Schriften zur sächsischen Geschichte und Volkskunde; Quellen und Materialien zur sächsischen Geschichte und Volkskunde; Bausteine aus dem Institut für Sächsische Geschichte und Volkskunde; Spurensuche. Geschichte und Kultur Sachsens. Projektleiter: Direktorium/Bereichsleiterinnen, Projektbearbeiter: Direktorium/Bereichsleiterinnen/wissenschaftliche Mitarbeiter.

Sachsen: weltoffen! Projektleiter: Direktorium/Bereichsleiterinnen, Projektbearbeiter: Ira Spieker, Kollegium des ISGV. 


\section{Projekte des Bereichs Geschichte}

Sächsische Biografie. Projektleiterin: Martina Schattkowsky, Projektbearbeiter: Martina Schattkowsky/Frank Metasch/Lutz Vogel.

Fürstinnenkorrespondenzen der Reformationszeit, Teil 1: Briefedition der Herzogin Elisabeth von Sachsen, Band 2 (1533-1534). Projektleiterin: Martina Schattkowsky, Projektbearbeiter: Jens Klingner.

Codex diplomaticus Saxoniae. Das Urkundenbuch der Stadt Dresden, Band 1. Projektleiter: Enno Bünz, Projektbearbeiterin: Ulrike Siewert.

Sächsisches Klosterbuch. Klöster, Stifte und Komtureien in Sachsen vor der Reformation. Projektleiter: Enno Bünz, Projektbearbeiterin: Sabine Zinsmeyer.

Zwischen Migration und Assimilation. Adel im sächsisch-böhmischen Grenzraum (16./17. Jahrhundert). Dissertationsprojekt. Projektleiterin: Martina Schattkowsky, Projektbearbeiter: Martin Arnold.

Für Gott und Vaterland - Patriotismus und Militärdienst in Sachsen 1806 bis 1866/67. Dissertationsprojekt. Projektleiter: Winfried Müller, Projektbearbeiter: Torsten Schwenke.

Finanz- und Geldgeschichte Sachsens im 18. Jahrhundert. Projektleiterin: Martina Schattkowsky, Projektbearbeiter: Frank Metasch.

Neues Archiv für sächsische Geschichte. Projektleiter: Karlheinz Blaschke/Enno Bünz/Winfried Müller/Martina Schattkowsky/Uwe Schirmer, Projektbearbeiter: Frank Metasch (Schriftleitung), Lutz Vogel (Rezensionen).

\section{Projekte des Bereichs Volkskunde}

Lebensgeschichtliches Archiv für Sachsen. Projektleiterin: Ira Spieker, Projektbearbeiter: Sönke Friedreich/Merve Lühr/Nadine Kulbe.

Visuelle Quellen zur Volkskultur in Sachsen. Das Bildarchiv des ISGV. Projektleiter: Andreas Martin/Ira Spieker, Projektbearbeiter: Andreas Martin/Hendrik Keller.

Fremde - Heimat - Sachsen: Vertriebene als Neubauern. Staatliche Integrationsmaßnahmen und individuelle Adaptionsstrategien. Projektleiterin: Ira Spieker, Projektbearbeiter: Ira Spieker/Sönke Friedreich/Uta Bretschneider/Nadine Kulbe/Ursula Schlude.

An der Elbe. Das Leben mit dem Fluss. Projektleiter: Andreas Martin/Ira Spieker, Projektbearbeiter: Andreas Martin.

Arbeiterfotografie als bildwissenschaftliches Ausstellungskonzept. Projektleiterin: Ira Spiecker, Projektbearbeiter: Wolfgang Hesse.

Zwischen Aufstieg und Krise. Städtische Identität und Selbstwahrnehmung in Plauen 1880 bis 1933. Projektleiterin: Ira Spieker, Projektbearbeiter: Sönke Friedreich. 
Neue Sichtweisen. Zum Aufleben einer Aussichtsturm-Begeisterung. Projektleiter: Andreas Martin/Ira Spieker, Projektbearbeiter: Andreas Martin.

Künstlersteinzeichnungen für Haus und Schule. Projektleiter: Winfried Müller, Projektbearbeiter: Winfried Müller.

Verordnete Nachbarschaften. Erinnerungskultur und Repräsentation im Grenzraum Sachsen, Schlesien und Böhmen seit dem Zweiten Weltkrieg. Projektleiterin: Ira Spieker, Projektbearbeiterinnen: Uta Bretschneider/Sarah Kleinmann.

Brigadebücher - Spiegel der Arbeitskultur in der DDR? (bis 3/2015)/Erinnern an die Arbeit im Kollektiv. Brigadeleben in der DDR und seine postsozialistischen Tradierungen. Dissertationsprojekt. Projektleiterin: Ira Spieker, Projektbearbeiterin: Merve Lühr.

Erschließung und Digitalisierung des Nachlasses „Adolf Spamer“. Projektleiterin: Ira Spieker, Projektbearbeiterin: Nadine Kulbe.

Volkskunde in Sachsen. Projektleiter: Ira Spieker/Sönke Friedreich, Projektmitarbeit: Mitarbeiterinnen und Mitarbeiter des Bereichs.

\section{Mitwirkung an Ausstellungen und Ausstellungskonzeptionen}

Zwischen Tradition und Modernität - Zeugnisse sächsischer Adelskultur (Arbeitstitel), Vorbereitung einer Dauerausstellung zur Geschichte des sächsischen Adels, Schloss Nossen. Konzeption und Organisation: Martina Schattkowsky.

Das Auge des Arbeiters. Arbeiterfotografie \& Kunst um 1930 (Stadtmuseum Dresden). Konzeption und Organisation: Wolfgang Hesse.

Fremdes Land. Neubauernfamilien in Sachsen (Wanderausstellung). Konzeption und Organisation: Ira Spieker/Uta Bretschneider.

\section{Tagungen und Workshops 2015}

Arbeiter | Kultur | Geschichte. Arbeiterfotografie der Weimarer Republik, Stadtmuseum Dresden, 27. und 28. März 2015. Konzeption und Organisation: Wolfgang Hesse.

Kurfürst August von Sachsen. Ein nachreformatorischer „Friedensfürst“ zwischen Territorium und Reich, Torgau, Schloss Hartenfels, Dresden, Residenzschloss, 9. bis 11. Juli 2015. Konzeption und Organisation: Winfried Müller/Martina Schattkowsky/ Dirk Syndram (Staatliche Kunstsammlungen Dresden).

Die Elbe - Fluss ohne Grenzen (1815-2015), Hamburg, Altonaer Museum für Kunst und Kulturgeschichte, 17. bis 19. September 2015. Konzeption und Organisation: Andreas Martin.

(Un)Gleiche Kurfürsten? Die Pfalzgrafen bei Rhein und die Herzöge von Sachsen im späten Mittelalter (1356-1517), Hauptstaatsarchiv Dresden, 8. und 9. Oktober 2015. 
Konzeption und Organisation: Jens Klingner/Benjamin Müsegades (Institut für Fränkisch-Pfälzische Geschichte und Landeskunde, Heidelberg).

Wissen - Wolle - Wandel. Merinoschafzucht und Agrarinnovation in Sachsen (18./19. Jahrhundert), Hauptstaatsarchiv Dresden, 5. und 6. November 2015. Konzeption und Organisation: Martina Schattkowsky/Ira Spieker, in Kooperation mit dem Sächsischen Staatsarchiv - Hauptstaatsarchiv Dresden.

1815: Europäische Friedensordnung - Mitteldeutsche Neuordnung. Die Neuordnung auf dem Wiener Kongress und ihre Folgen für den mitteldeutschen Raum, Merseburg, Ständehaus, 5. bis 7. November 2015. Konzeption und Organisation: Ulrike Höroldt/ Andreas Erb/Hans-Werner Hahn/Frank-Lothar Kroll/Winfried Müller.

\section{Publikationen 2015}

Neues Archiv für sächsische Geschichte, hrsg. von Karlheinz Blaschke/Enno Bünz/ Winfried Müller/Martina Schattkowsky/Uwe Schirmer. Redaktion: Frank Metasch (Schriftleitung), Lutz Vogel (Rezensionen), Band 86 (2015), Neustadt a. d. Aisch: Verlag Ph. C. W. Schmidt.

Volkskunde in Sachsen, hrsg. vom Institut für Sächsische Geschichte und Volkskunde e. V. Schriftleitung: Ira Spieker/Sönke Friedreich, unter Mitarbeit von Uta Bretschneider/Nadine Kulbe/Merve Lühr, Band 27 (2015), Dresden: Thelem Universitätsverlag.

Schriften zur sächsischen Geschichte und Volkskunde, hrsg. von Enno Bünz/Winfried Müller/Martina Schattkowsky/Ira Spieker, Leipzig: Leipziger Universitätsverlag:

Band 48: Christian Heinker, Die Bürde des Amtes - die Würde des Titels. Der kursächsische Geheime Rat im 17. Jahrhundert.

Band 50: Enno Bünz/Hartmut Kühne (Hg.), Alltag und Frömmigkeit am Vorabend der Reformation in Mitteldeutschland. Wissenschaftlicher Begleitband zur Ausstellung „Umsonst ist der Tod“.

Band 51: Olav Heinemann, Das Herkommen des Hauses Sachsen. Genealogischhistoriographische Arbeit der Wettiner im 16. Jahrhundert.

Band 52: Swen Steinberg, Unternehmenskultur im Industriedorf. Die Papierfabriken Kübler \& Niethammer in Sachsen (1856-1956).

Quellen und Materialien zur sächsischen Geschichte und Volkskunde, hrsg. von Enno Bünz/Winfried Müller/Martina Schattkowsky/Ira Spieker, Leipzig: Leipziger Universitätsverlag:

Band 5: Peter Wiegand, Der päpstliche Kollektor Marinus de Fregeno († 1482) und die Ablasspolitik der Wettiner. Quellen und Untersuchungen.

\section{Online-Publikationen (Weiterfübrung)}

Digitales Historisches Ortsverzeichnis von Sachsen (DHOV). Verantwortlich: Ulrike Siewert, URL: http://hov.isgv.de/orte/

Repertorium Saxonicum. Verantwortlich: Ulrike Siewert, URL: http://www.isgv.de/ repsax/ 
Der Codex diplomaticus Saxoniae im Internet. Verantwortlich: Ulrike Siewert, URL: http://codex.isgv.de/

Sächsische Biografie. Verantwortlich: Martina Schattkowsky, URL: http://saebi.isgv. de/

Das Auge des Arbeiters. Untersuchungen zur proletarischen Amateurfotografie am Beispiel Sachsens. Verantwortlich: Wolfgang Hesse, URL: www.arbeiterfotografiesachsen.de/

Fremde - Heimat - Sachsen: Vertriebene als Neubauern. Staatliche Integrationsmaßnahmen und individuelle Adaptionsstrategien. Verantwortlich: Ira Spieker/Sönke Friedreich/Uta Bretschneider, URL: www.neubauern-sachsen.de/

Sachsen.digital: Interdisziplinäre Wissensplattform zur Geschichte, Kultur und Landeskunde Sachsens, gemeinsames Internetportal der Sächsischen Landesbibliothek Staats- und Universitätsbibliothek Dresden und des ISGV. Verantwortlich: Martina Schattkowsky/Ira Spieker/Ludwig Felber/Michael Schmidt, URL: www.sachsendigi tal.de/

Lebensgeschichtliches Archiv für Sachsen. Verantwortlich: Ira Spieker, URL: http:// lga.isgv.de/

Visuelle Quellen zur Volkskultur in Sachsen. Das Bildarchiv des ISGV. Verantwortlich: Andreas Martin, URL: http://bild.isgv.de/

Sachsen: weltoffen! Verantwortlich: Ira Spieker, URL: http://www.isgv.de/aktuelles/ sachsen-weltoffen/ 